\title{
Increased KIAA0101 gene expression associated with poor prognosis in breast cancer
}

\author{
Jiuyang Liu", Yukun He”, Chengxin Li, Rui Zhou, Qianqian Yuan, Jinxuan Hou, Gaosong Wu \\ Department of Breast \& Thyroid Surgery, Zhongnan Hospital of Wuhan University, Wuhan, China \\ Contributions: (I) Conception and design: G Wu, J Hou, J Liu; (II) Administrative support: G Wu; (III) Provision of study materials or patients: J \\ Hou, J Liu, Y He; (IV) Collection and assembly of data: J Liu, Y He; (V) Data analysis and interpretation: J Liu, Y He, C Li, Q Yuan, R Zhou; (VI) \\ Manuscript writing: All authors; (VII) Final approval of manuscript: All authors. \\ \#These authors contributed equally to this work. \\ Correspondence to: Prof. Jinxuan Hou; Gaosong Wu. Department of Breast \& Thyroid Surgery, Zhongnan Hospital of Wuhan University, Wuhan \\ 430071, China. Email: jhou@whu.edu.cn; wugaosongtj@163.com.
}

Background: Breast cancer (BC) has long been a major death threat facing women worldwide. With the development of comprehensive treatment methods, the prognosis of BC was improved but still unsatisfactory. This study was aimed to identify the key genes in $\mathrm{BC}$ tumorigenesis and investigate potential prognostic predictors.

Methods: Differential expression genes were analyzed in TCGA BRCA dataset using Genevestigator software. The expression profile of target gene was explored, and the correlations between selected genes with important clinical parameters were evaluated as well. The prognostic values of target genes were also carried out through Kaplan-Meier plotter OncoLnc and BC gene-expression miner.

Results: KIAA0101 gene was selected for further analysis from the differential expression genes identified. At both mRNA and protein levels, the expression of KIAA0101 in BC was higher than that in normal tissues. Further analysis indicated that overexpression of KIAA0101 was significantly correlated with worse clinical outcome parameters. KIAA0101 was highly expressed in older patients, in the luminal group, and in patients with advanced stages. Moreover, BC patients with elevated KIAA0101 expression had worse overall survival (OS), relapse-free survival (RFS), distant metastasis-free survival (DMFS) and disease-free survival (DFS).

Conclusions: Taken together, KIAA0101 could be considered as a diagnostic biomarker or predictor for $\mathrm{BC}$ prognosis.

Keywords: KIAA0101; breast cancer (BC); prognosis

Submitted Oct 15, 2020. Accepted for publication Jul 14, 2021.

doi: $10.21037 /$ tcr-20-3064

View this article at: https://dx.doi.org/10.21037/tcr-20-3064

\section{Introduction}

For long breast cancer (BC) has been the most common neoplasm diagnosed amongst women and a major cause of cancer-related deaths worldwide (1). BC was also the most common cancer in Chinese women, especially in urban areas (2). In recent years, the morbidity and mortality were markedly decreased in BC patients because of employing early diagnosis and comprehensive treatment strategies including surgery, chemotherapy, radiotherapy, endocrine agents, and biological targeting agents. However, the prognosis of BC is still unsatisfactory. Screening and identifying novel key molecules in BC development and progression may provide a promising solution. With the development of integrative bioinformatics, high-throughput gene expression analysis is commonly used to find the differentially expressed genes involved in BC tumorigenesis (3).

KIAA0101 is a $15-\mathrm{kDa}$ protein containing a conserved proliferating cell nuclear antigen (PCNA)-binding motif, which was first identified as a major partner of 
PCNA in yeast two hybrid screening (4,5). KIAA0101 was subsequently designed as PCNA-associated factor (PAF15) (5), and was also named as L5 (dysregulated in non-small-cell lung cancer) (6), overexpressed in anaplastic thyroidcarcinoma-1 (OEATC-1) (7), non-structural protein 5A (NS5A)-transactivated protein 9 (NS5ATP9) (8) and PCNA clamp associated factor (PCLAF) (9). Herein, KIAA0101 will be studied throughout this study.

KIAA0101 has been reported to be involved in DNArepair process by mediating the interaction with DNA synthesis polymerase (10). Aberrant expression of KIAA0101 was found in various cancers including nonsmall cell lung cancer (6), anaplastic thyroid carcinoma (7), hepatocellular carcinoma (11), BC (12) and ovarian cancer (13). Overexpression of KIAA0101 was observed in Ki67-positive BC tumors, suggesting association between overexpression of KIAA0101 and increased chances of relapse and worse survival rates (12). However, it was controversial that KIAA0101 depletion caused centrosome amplification while KIAA0101 was important in the UV damage response, indicating that KIAA0101 might function as a tumor suppressor gene. Therefore, the utility of KIAA0101 as a biomarker or a prognostic factor for BC is currently unclear.

The present study aims to identify the differential expression genes upregulated in BC tissues by using integrative bioinformatics. Further analysis is focused on KIAA0101 gene. In addition to evaluating the expression level, we also discussed the significance of KIAA0101 gene expression based on clinical indicators and survival data in several large online databases.

We present the following article in accordance with the REMARK reporting checklist (available at https://dx.doi. org/10.21037/tcr-20-3064).

\section{Methods}

\section{Identification of differential expression genes}

The differential expression genes between BC tissues and adjacent normal tissues were identified by using Genevestigator software, which contains a comprehensive collection of public microarrays and RNA-Seq study results. The Cancer Genome Atlas (TCGA) breast cancer (BRCA) dataset (1,200 samples, homo sapiens) was chosen for further analysis, and infiltrating ductal and lobular carcinoma was compared with adjacent breast tissue. The study was conducted in accordance with the Declaration of Helsinki (as revised in 2013).

\section{Oncomine analysis}

The mRNA expression level of KIAA0101 was analyzed in tumor tissues and matched normal tissues based on the Oncomine Platform (www.oncomine.org). The analysis was conducted using the following filters: gene, KIAA0101; analysis type, differential analysis, cancer $v s$. normal analysis; cancer type, BC; and data type, mRNA. In the study, twofold change, $\mathrm{P}$ value $=1 \mathrm{E}-4$ and top $10 \%$ gene rank as threshold was selected. The statistical methods and values were performed automatically by the Oncomine Platform.

\section{Gene Expression Profiling Interactive Analysis (GEPIA) dataset}

GEPIA (http://gepia.cancer-pku.cn/) is a newly developed interactive web server, which employs a standard processing pipeline for analyzing the RNA sequencing expression data from TCGA and the Genotype-Tissue Expression (GTEx) projects (9,736 tumors and 8,587 normal samples). The present study used GEPIA to perform differential expression analysis for KIAA0101 in tumor and normal samples, as well as profiling according to cancer types or pathological stages.

\section{Breast cancer gene-expression miner (bcGenExMiner)}

The expression of PCLAF (another gene symbol for KIAA1199) mRNA in different subtypes of BC was analyzed using the bcGenExMiner v4.4 (http://bcgenex. centregauducheau.fr/BC-GEM). The relationship between KIAA0101 gene expression and clinical characteristics in BC patients were compared using target gene analysis. The characteristics includes age, estrogen receptor (ER) status, progesterone receptor (PR) status, human epidermal growth factor receptor-2 (HER-2) status, histological types, p53 status, Scarff-Bloom-Richardson (SBR) and the Nottingham Prognostic Index (NPI). A Welch's test is performed (along with Dunnett-Tukey-Kramer's tests for pairwise comparison when appropriate).

\section{UALCAN cancer database}

UALCAN is a comprehensive, user-friendly and interactive web resource for analyzing cancer OMICS data (http:// ualcan.path.uab.edu). Protein expression analysis could be performed by UALCAN using data from Clinical Proteomic Tumor Analysis Consortium (CPTAC) 
A

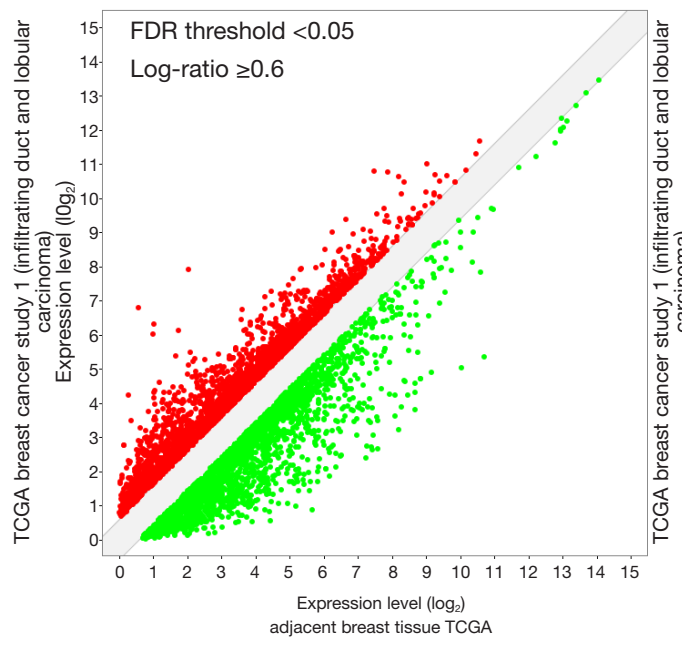

B

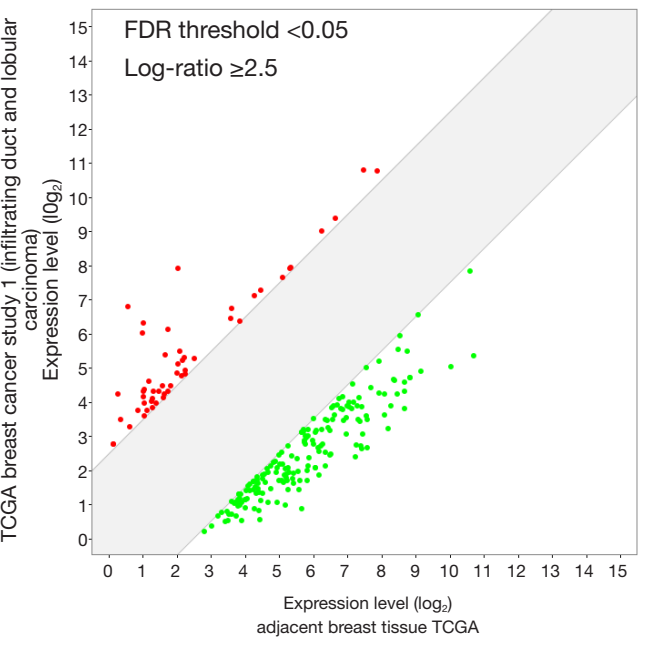

Figure 1 The differential expression genes were identified from TGCA BRCA database (1,200 samples, homo sapiens) using GENEVESTIGATOR $^{\circledR}$. (A) Genevestigator analysis of KIAA0101 with FDR P value less than 0.05 and log-ratio above 0.6 ; (B) the logratio was increased up to 2.5 to narrow the target gene range.

Confirmatory/Discovery dataset. The present study aims to explore the correlation between KIAA0101 protein expression and clinical indicators in $\mathrm{BC}$.

\section{Kaplan-Meier Plotter}

Kaplan-Meier Plotter is an online tool for discovery and validation of survival biomarkers (http://kmplot.com/ analysis). To analyze the prognostic value of KIAA0101 gene, the patient samples are split into two groups according to the median expression of the proposed gene (high expression $v s$. low expression). There are two probes for KIAA0101 in Kaplan-Meier Plotter (NS5ATP9, 211713_x_at probe and KIAA0101, 202503_s_at probe). The study point includes overall survival (OS), relapse-free survival (RFS), distant metastasis free survival (DMFS) and post progression survival (PPS). The two patient cohorts are compared by a Kaplan-Meier survival plot, and the hazard ratio with $95 \%$ confidence intervals and log-rank $\mathrm{p}$ value are calculated.

\section{OncoLnc}

OncoLnc is an online tool linking TCGA survival data to mRNA, miRNA, or lncRNA expression levels (http://www. oncolnc.org/). A total of 1,006 BC patients with survival data is included in mRNA datasets of OncoLnc.

\section{PrognoScan}

PrognoScan includes public microarray datasets with clinical annotation of gene expression and prognosis from Gene Expression Omnibus (GEO), Array Express and individual laboratory websites (http://www.prognoscan.org/). PrognoScan provides a powerful platform for evaluating potential tumor markers and therapeutic targets, which searches the relation between gene expression and patient prognosis across a large collection of publicly available cancer microarray datasets. The correlation between KIAA0101 expression and survival data in BCs was analyzed by PrognoScan database.

\section{Results}

\section{Identification of differentially expressed genes from TCGA BRCA database}

The differential expression genes were identified from TCGA BRCA database (1,200 samples, homo sapiens) using GENEVESTIGATOR ${ }^{\circledR}$. Comparing adjacent normal breast tissue if adjacent breast tissue is normal tissue, a total of 5041 differential expressed genes were identified, including 2,779 upregulated and KI downregulated as shown in Figure $1 A$ and https://cdn.amegroups.cn/static/ public/TCR-20-3064-1.xlsx (FDR <0.05). For restricting the target genes, the log-ratio was increased up to 2.5 from 
0.6. 229 genes (51 upregulated and 178 downregulated, https://cdn.amegroups.cn/static/public/TCR-20-3064-2. pdf) were obtained for further investigation (Figure 1B). We focus on KIAA0101 as which functions as a regulator of centrosome number in $\mathrm{BC}$ and plays a key role in recruiting DNA replicative polymerase by double monoubiquitination. The $\log$-ratio of KIAA0101 is 2.81 and the $\mathrm{P}$ value of KIAA0101 is $5.58 \mathrm{E}-38$.

\section{Increased expression of KIAA0101 gene in various malignant tumors}

The expression of KIAA0101 gene was evaluated in 20 malignant tumors using the Oncomine database and 33 tumors with GEPIA dataset. Increased expression level of KIAA0101 (red label) was observed in most malignant tumors versus normal tissues, including BC (Figure $2 A, 2 B$ ). Further analysis using TCGA BRCA dataset revealed that KIAA0101 was significantly higher expressed in all kinds of BC histological types, compared with the corresponding normal tissues (Figure 2C), which was in consistent with the results obtained from Curtis breast dataset (Figure 2D) and GEPIA dataset (Figure 2E). In addition, GEPIA violin plot of KIAA0101 gene expression by pathological stages based on the TCGA clinical annotation showed elevated expression levels significantly associated with advanced TNM stage (Figure 2F).

\section{KIAA0101 gene expression according to different clinical characteristics in $B C$}

The relationship between KIAA0101 gene expression and clinical indicators in $\mathrm{BC}$ patients were compared by using the bc-GenExMiner v4.4 online tool (as shown in Figure 3). The expression of KIAA0101 was significantly higher in younger group than that in older group (Figure $3 A, 3 B$ ). Among the histological types, infiltrating ductal carcinoma showed the most elevated expression (Figure 3C). ER and PR status were negatively correlated with KIAA0101 expression, while HER-2 status was positively associated with KIAA0101 expression (Figure 3D-3F). In addition, KIAA0101 expression was markedly elevated in basallike subtype and triple-negative breast cancer (TNBC) with respect to non-basal-like subtype and non-TNBC patients (Figure 3G-37). Overexpression of KIAA0101 was observed in BC patients with positive nodal status $(\mathrm{N}+$, Figure $3 K)$. Mutated p53 status accompany with higher level of KIAA0101 than the wild type p53 status in both immunohistochemistry and sequence-base analysis (Figure $3 L, 3 M$ ). To evaluate tubule formation, nuclear characteristics of pleiomorphism, and mitotic index, a histological grade, the Scarff-Bloom-Richardson (SBR) is induced. And the Nottingham Prognostic Index (NPI) has been defined to stratify patients into additional prognostic groups according to tumor size, lymph node stage, and tumor grade. The data revealed that advanced SBR grade and NPI showed higher KIAA0101 expression (Figure 3N,3O). Taken together, increased expression of KIAA0101 is significantly correlated with worse outcome indicators $(\mathrm{P}<0.01)$.

\section{KIAA0101 protein expression according to different clinical parameters in $B C$}

Further analysis was performed to evaluate the protein expression of KIAA0101 in BC using UALCAN web tool with CPTAC dataset, including total protein and phosphoprotein (Figure 4). The protein level of KIAA0101 expression was significantly higher in tumors than that in normal tissues $(\mathrm{P}<0.001$, Figure $4 A, 4 G)$. In consistent with gene expression, younger patients have higher KIAA0101 protein expression compared with older patients $(\mathrm{P}<0.001$, Figure $4 B, 4 H)$. Due to insufficient sample sizes, the difference among tumor histology groups indicated limited significance (Figure 4C,4I). The luminal group showed the most abundant KIAA0101 protein expression than the Her2 positive group and the TNBC group, which is different from gene level (Figure 4D,47). For individual cancer stage, KIAA0101 was highly expressed in patients with advanced stages as the same with gene level (Figure 4E,4K). Moreover, KIAA0101 protein was detected in various malignant tumors (Figure 4F,4L).

\section{Increased KIAA0101 expression correlated with poor prognosis in BC patients}

The prognostic value of KIAA0101 was evaluated in several databases with different cohorts (Figure 5). Employing two different probes, the results from the Kaplan Meier plotter showed that high level of KIAA0101 was correlated with worse OS ( $\mathrm{P}=0.00017$, Figure $5 A ; \mathrm{P}=0.00039$, Figure $5 E)$, worse RFS $(\mathrm{P}=4.6 \mathrm{e}-6$, Figure $5 B$; $\mathrm{P}<1 \mathrm{E}-6$, Figure $5 F)$, worse DMFS $(\mathrm{P}=3.8 \mathrm{e}-5$, Figure $5 C ; \mathrm{P}=0.00064$, Figure $5 G)$ and worse PPS $(\mathrm{P}=0.00083$, Figure $5 D)$, comparing with low expression level patients. There was no significant difference for the PPS using 202503_s_at probe $(\mathrm{P}=0.23$, Figure $5 H)$. 
A

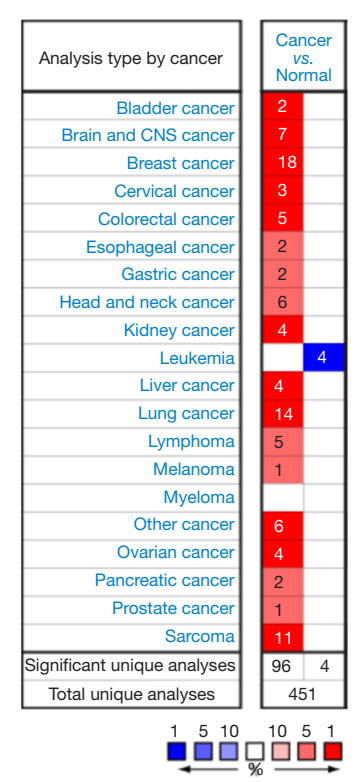

B

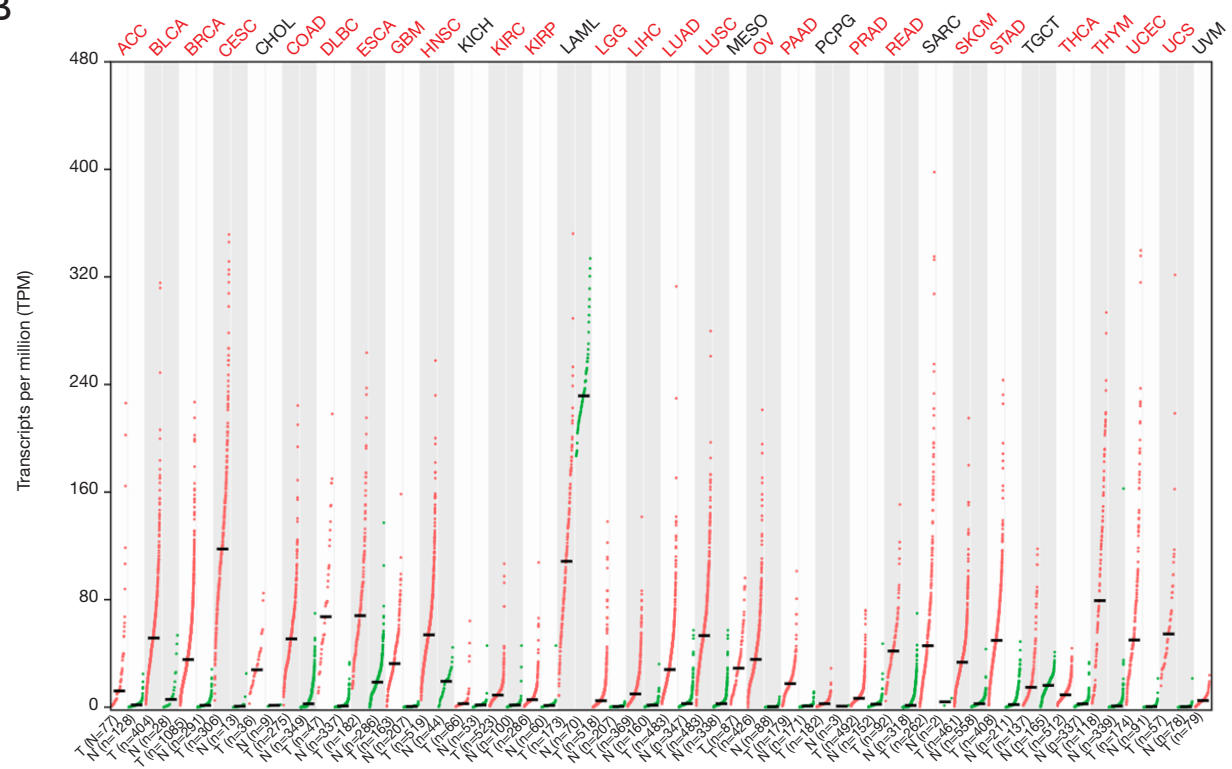

C

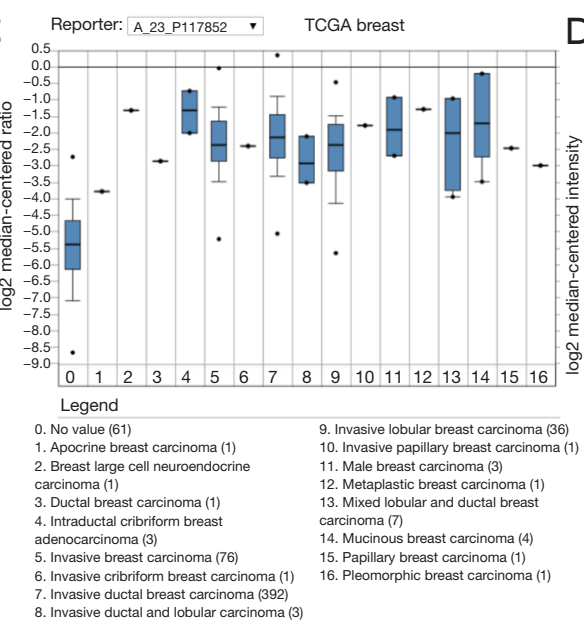

D Reporter: ILMN_1732150 - Curtis breast

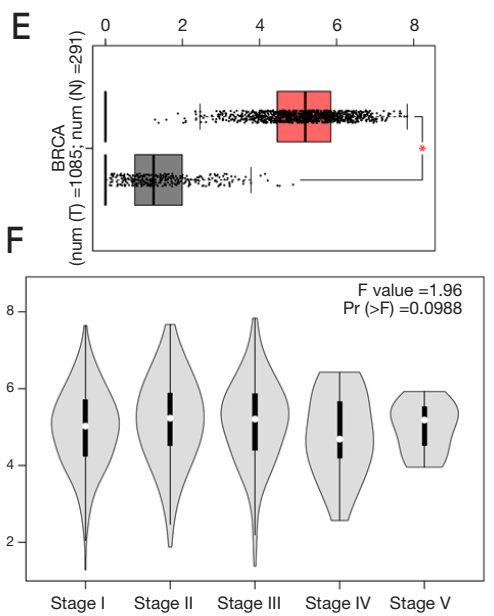

Figure 2 Increased expression of KIAA0101 gene in various malignant tumors. (A) Expression of KIAA0101 gene in 20 malignant tumors versus corresponding normal tissues using the Oncomine database. Statistically significant increased and decreased levels of KIAA0101 gene were labeled red and blue, respectively, with the number of datasets $(\mathrm{P}<0.05)$. (B) The KIAA0101 gene expression profile across all tumor samples and paired normal tissues from GEPIA dataset. Each dot represents expression of samples. Red, tumor; green, normal. (C,D) Box plot comparing KIAA0101 expression in BC patients and normal individuals derived from TCGA BRCA dataset (C) and Curtis breast dataset (D), respectively. · stands for the Outlier values. (E) Higher level of KIAA0101 gene expression in breast cancer compared with matched normal tissue (GEPIA). *, P<0.05. (F) GEPIA violin plot of KIAA0101 gene expression by pathological stages based on the TCGA clinical annotation. BC, breast cancer.

The data from OncoLnc indicated that high expression of KIAA0101 revealed worse prognosis than low expression group ( $\mathrm{P}=0.0189$, Figure 5I). Moreover, Correlation between expression of KIAA0101 and OS as well as metastatic RFS was verified in a large population by using the bc-GenExMiner database (OS: $\mathrm{P}<0.0001$, Figure 57; metastatic RFS: $\mathrm{P}<0.0001$, Figure $5 K$ ). Furthermore, the PrognoScan database showed that overexpression of KIAA0101 was significantly associated with worse OS, RFS, DMFS, DSS, and DFS (Table 1). 


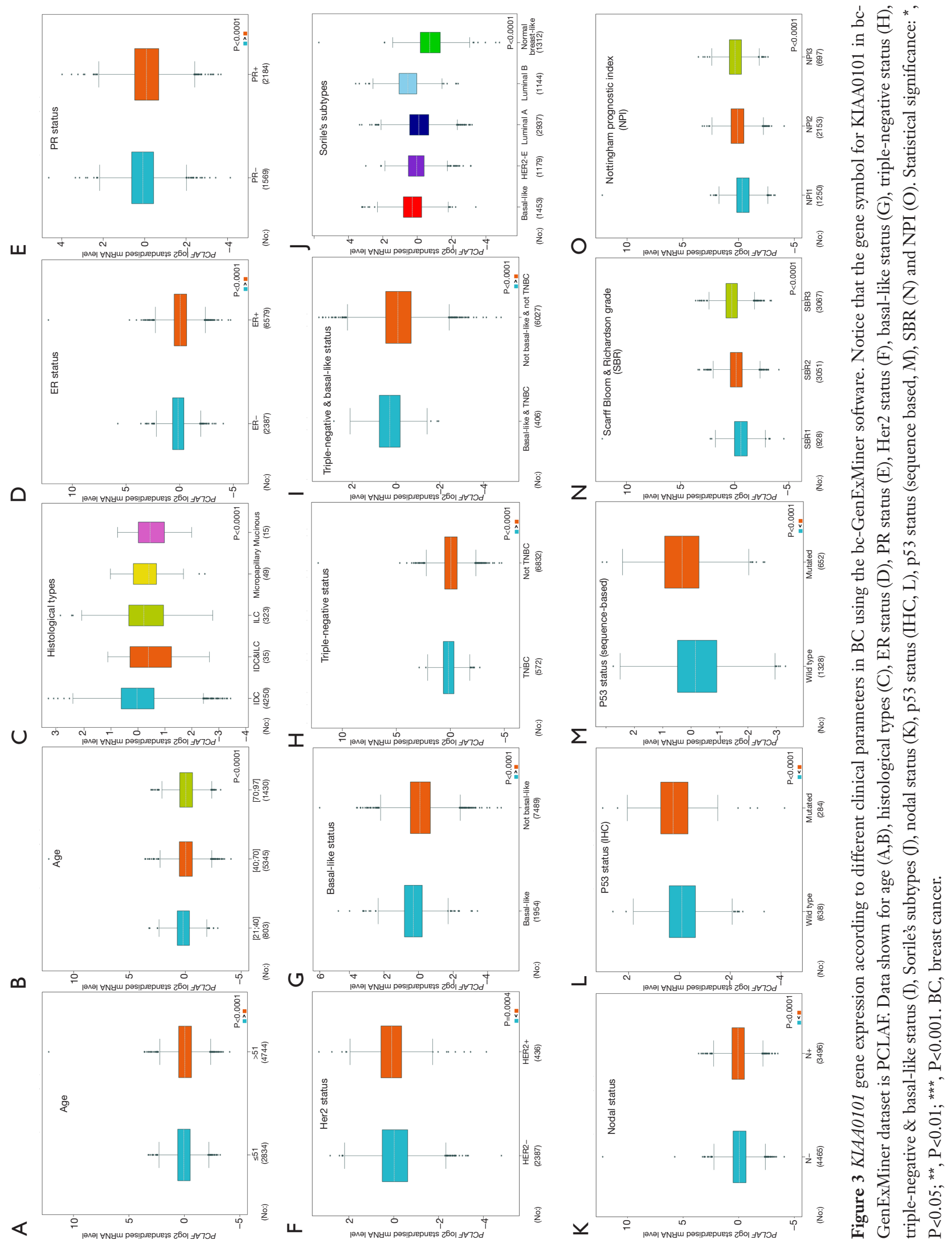


A

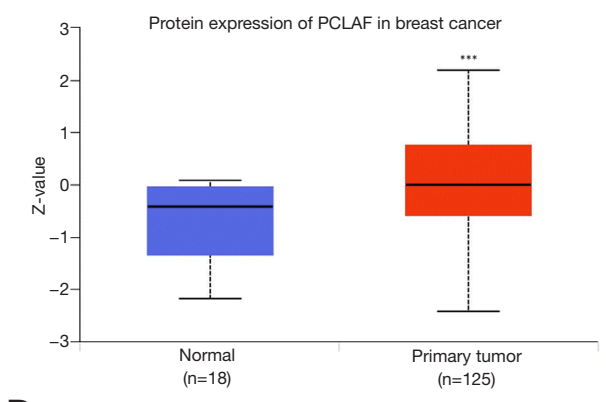

D

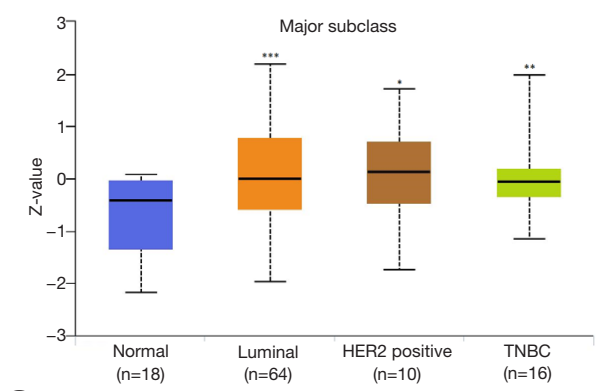

G

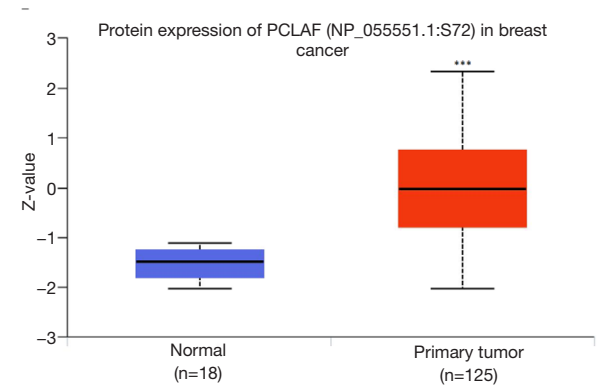

J

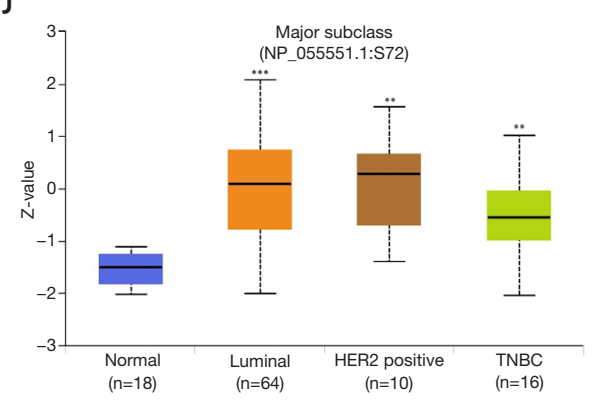

B

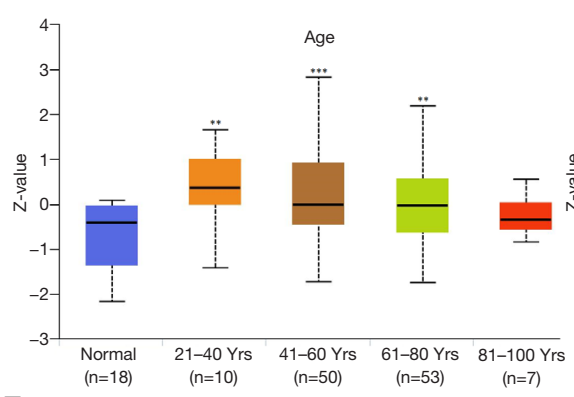

$E$

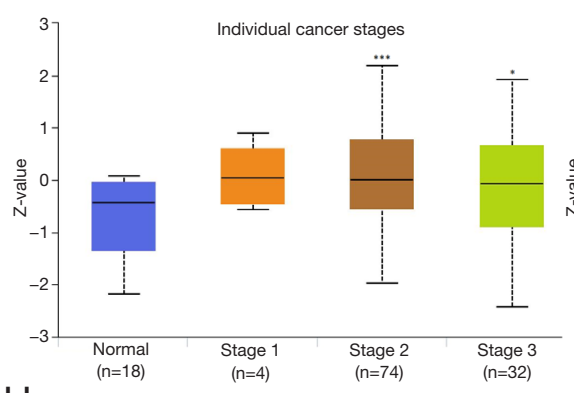

$\mathrm{H}$

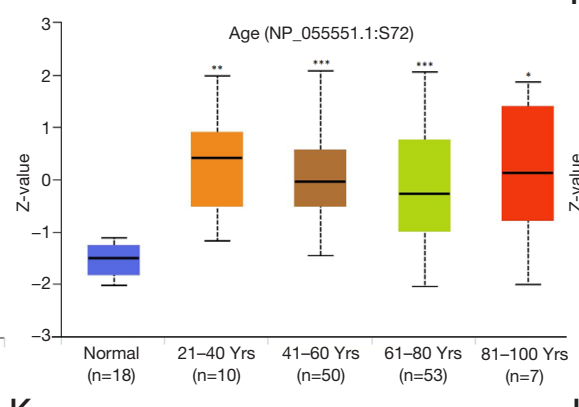

K

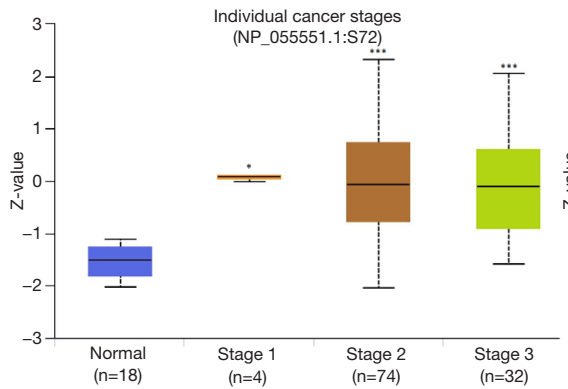

C

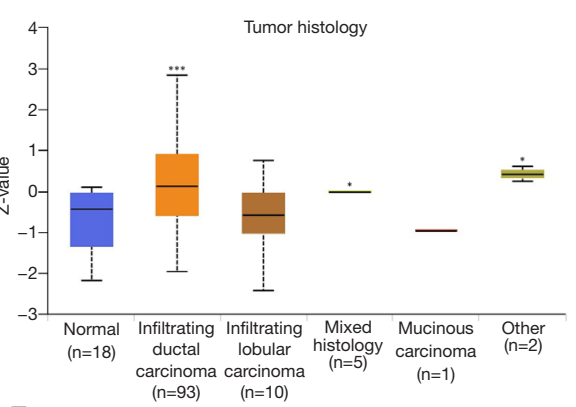

$\mathrm{F}$
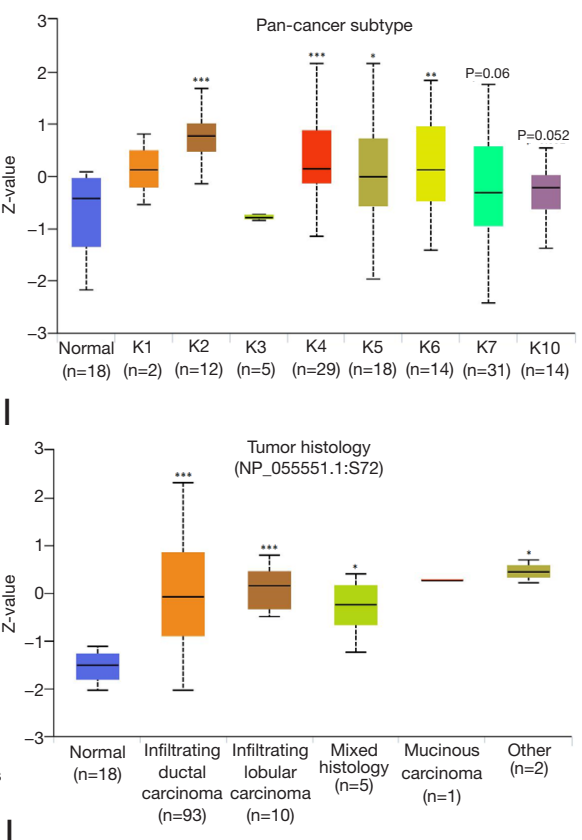

L

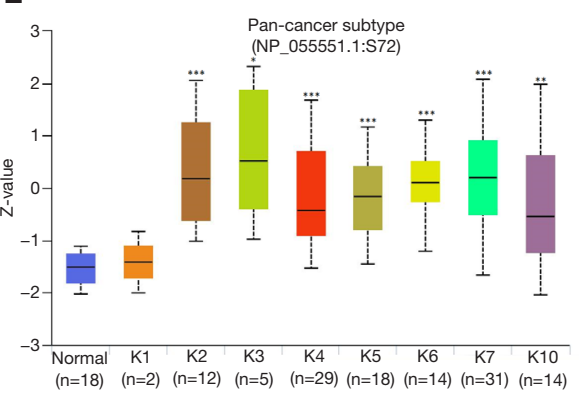

Figure 4 KIAA0101 protein expression according to different clinical parameters in BC using UALCAN web tool. Notice that the gene name for KIAA0101 in CPTAC dataset is PCLAF. The protein level of KIAA0101 expression was analyzed both in total-protein database (A-F) and phosphor-protein database (G-L). Data shown for total evaluation (A,G), age (B,H), tumor histology (C,I), major subclass (D,J), individual cancer stages $(\mathrm{E}, \mathrm{K})$ and pan-cancer subtypes (F,L). Statistical significance: * $\mathrm{P}<0.05$; **, $\mathrm{P}<0.01$; ${ }^{* * *}, \mathrm{P}<0.001$. BC, breast cancer. 


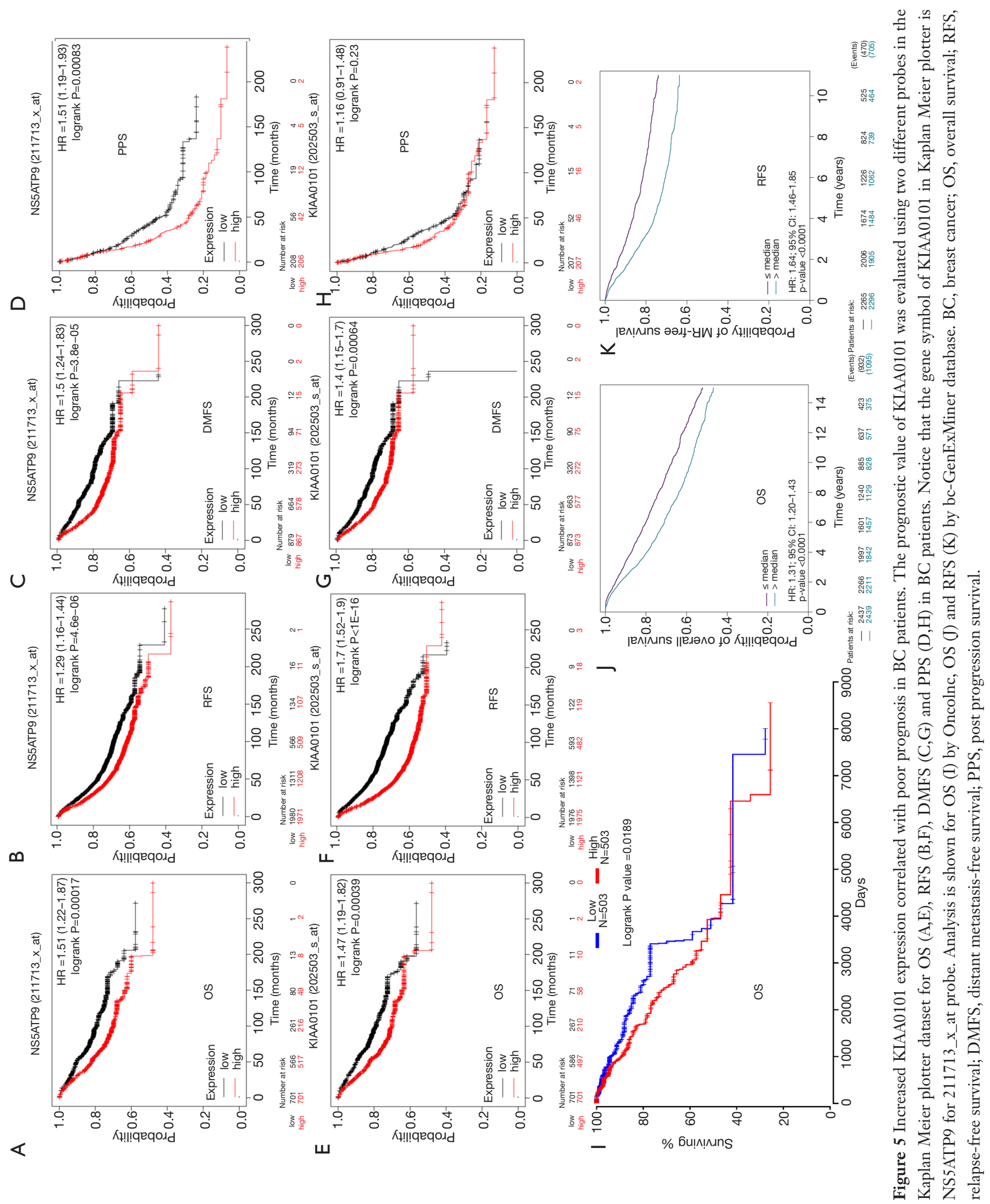


Table 1 The relation between KIAA0101 expression and BC patient prognosis across a large collection of cancer microarray datasets

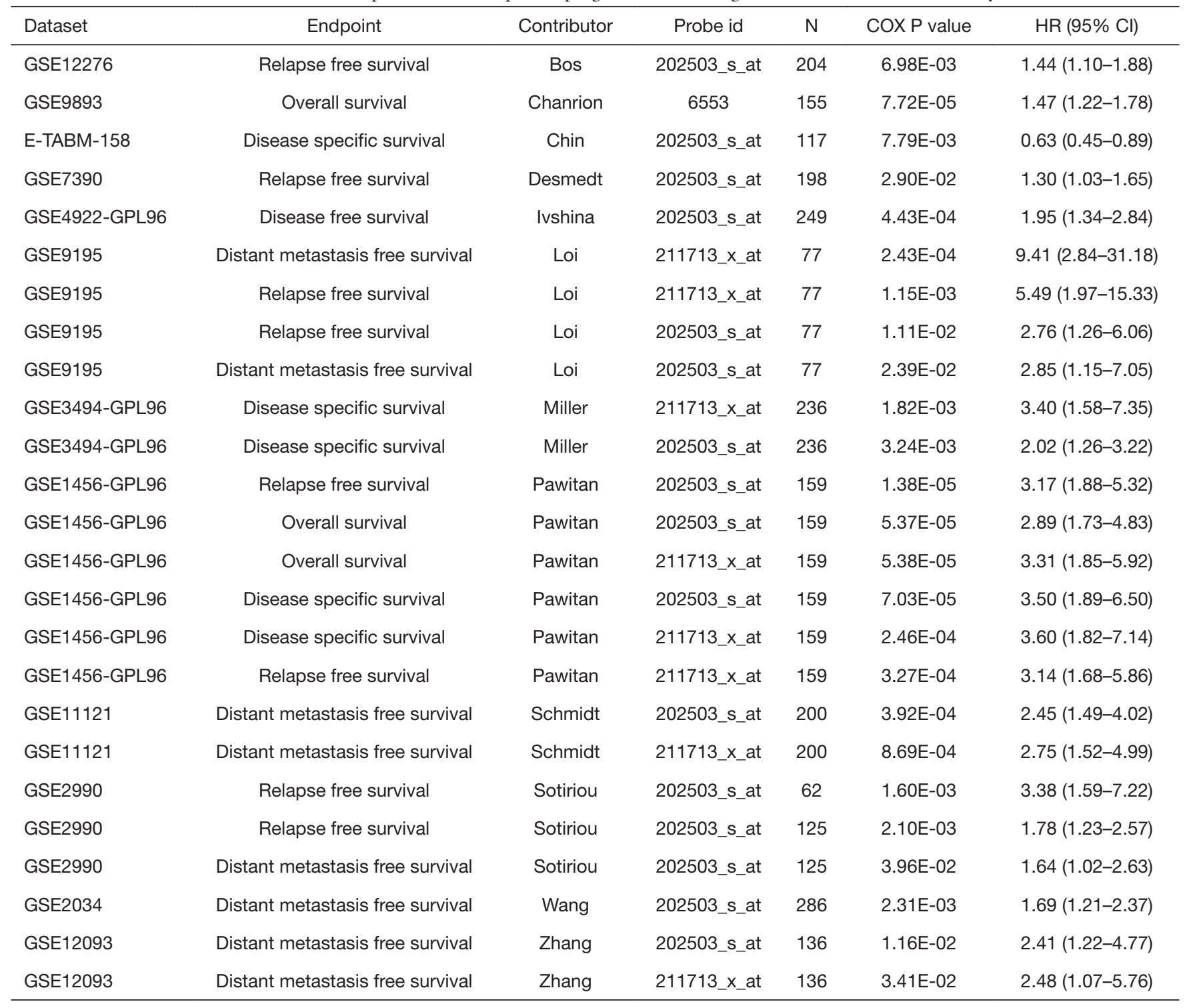

\section{Discussion}

BC has always been a major death threat facing women worldwide. Although utilizing traditional treatments (surgery, chemotherapy, radiotherapy and endocrine therapy) and extending novel treatment strategies (target therapy and immune-therapy) have already improved the outcome of BC patients, it is still looking forward to a better prognosis. Comprehensive meta-analysis of microarray data, combined with node analysis and functional analysis, is an effective strategy to analyze key caner-associated genes and signaling pathways (14).
In this study, the differential gene expression of breast carcinoma comparing adjacent normal tissues was analyzed in order to elucidate the abnormal key genes involved in $\mathrm{BC}$ tumorigenesis and progression. Through the analysis of TCGA BRCA database, 5,041 differentially expressed genes were identified, including 2,779 upregulated and 2,262 downregulated. In order to limit the target gene, the log-ratio was increased from 0.6 to 2.5 , and 229 genes (51 up-regulated and 178 down regulated) were obtained for further study. By reviewing the literatures, we focused on the KIAA0101 gene. The expression of KIAA0101 is 
highly dynamic in cell cycle, and it is most abundant in $\mathrm{S}$ phase (15) and G2 phase (16). As KIAA0101 expression may contribute to DNA repair due to DNA damage caused by UV irradiation, upregulation of KIAA0101 expression could enhance the cell survival (17). Accumulated evidence suggests that KIAA0101 is overexpressed in cancer (18). The increased level of KIAA0101 was positively correlated with the early recurrence and poor prognosis of liver cancer (11), lung cancer (19) and gastric cancer (20).

In BC, Kais et al. found that KIAA0101 could regulate the number of centrosomes by physical interaction with BC associated genes (BRCA1), in which KIAA0101 and BRAC1 together prevented centrosome re-duplication within the same cell cycle (12). KIAA0101 gene was identified using cross-species comparative genomics analysis of progression from pre-invasive ductal carcinoma in situ (DCIS) to invasive ductal carcinoma (IDC), that may be involved in tumorigenesis and in the processes of invasion and progression of disease (21). Shubbar et al. (22) analyzed a panel of 80 breast tumors by IHC to show that the protein level of KIAA0101 did not correlate with any clinicopathological parameter and had no influence on disease-specific survival (DSS). Recent study showed that KIAA0101 knockdown suppressed the cell proliferation and cell cycle progression by promoting the formation of $\mathrm{p} 53$ / Sp1 complex in BC (23). However, the utility of KIAA0101 as a biomarker for $\mathrm{BC}$ is still uncertain.

In this study, we analyzed the expression profile of KIAA0101 in Oncomine database and GEPIA database, showed that higher level of KIAA0101 mRNA was detected in $\mathrm{BC}$ tissues than that in matched normal tissue. The similar results were observed for protein expression profile of KIAA0101 using UALCAN cancer database. It is indicated that KIAA0101 might serve as a biomarker for distinguishing $\mathrm{BC}$ and normal tissue. Moreover, the relationship between KIAA0101 expression and clinical indicators in BC patients were evaluated both in mRNA (bc-GenExMiner online tool) and protein level (UALCAN). It is discovered that (I) age status was negatively correlated with KIAA0101 level as the same as in liver cancer (8); (II) KIAA0101 abundance is correlated with malignancy grade as in astrocytoma (24) and hepatocellular carcinoma (11); (III) KIAA0101 expression was negatively correlated with ER and PR status, while positively associated with HER2 status; Overexpression of KIAA0101 was observed in BC patients with basal-like subtype, TNBC, positive nodal status, advanced SBR grade and NPI. Taking the data together, overexpression of KIAA0101 is significantly correlated with worse outcome parameters. We further explored the prognostic value of KIAA0101 in BC using the Kaplan-Meier Plotter, OncoLnc and PrognoScan databases. Patients with increased KIAA0101 significantly correlated with worse OS, RFS, DMFS and DFS. These findings collectively elucidated that the expression of KIAA0101 might be a predictive biomarker for prognosis of $\mathrm{BC}$.

In conclusion, to our knowledge, it is the first time to use integrative bioinformatics to explore the expression profile and prognostic significance of KIAA0101 in BC. KIAA0101 was overexpressed in $\mathrm{BC}$ and correlated with a worse survival. It is suggested that KIAA0101 could be considered as a diagnostic biomarker or predictor for BC prognosis. Further experiments are essential to elucidate the value of KIAA0101 in BC.

\section{Acknowledgments}

Funding: This work was supported by the Fundamental Research Funds for the Central Universities of Ministry of Education of China (No. 2042019kf0141), and Zhongnan Hospital of Wuhan University Science, Technology and Innovation Seed Fund (WJ2019H027; WJ2019H015).

\section{Footnote}

Reporting Checklist: The authors have completed the REMARK reporting checklist. Available at https://dx.doi. org/10.21037/tcr-20-3064

Conflicts of Interest: All authors have completed the ICMJE uniform disclosure form (available at https://dx.doi. org/10.21037/tcr-20-3064). The authors have no conflicts of interest to declare.

Ethical Statement: The authors are accountable for all aspects of the work in ensuring that questions related to the accuracy or integrity of any part of the work are appropriately investigated and resolved. The study was conducted in accordance with the Declaration of Helsinki (as revised in 2013). Institutional ethical approval and informed consent were waived.

Open Access Statement: This is an Open Access article distributed in accordance with the Creative Commons Attribution-NonCommercial-NoDerivs 4.0 International License (CC BY-NC-ND 4.0), which permits the noncommercial replication and distribution of the article with 
the strict proviso that no changes or edits are made and the original work is properly cited (including links to both the formal publication through the relevant DOI and the license). See: https://creativecommons.org/licenses/by-nc-nd/4.0/.

\section{References}

1. Bray F, Ferlay J, Soerjomataram I, et al. Global cancer statistics 2018: GLOBOCAN estimates of incidence and mortality worldwide for 36 cancers in 185 countries. CA Cancer J Clin 2018;68:394-424.

2. Chen W, Sun K, Zheng R, et al. Cancer incidence and mortality in China, 2014. Chin J Cancer Res 2018;30:1-12.

3. Russo G, Zegar C, Giordano A. Advantages and limitations of microarray technology in human cancer. Oncogene 2003;22:6497-507.

4. Warbrick E. A functional analysis of PCNA-binding peptides derived from protein sequence, interaction screening and rational design. Oncogene 2006;25:2850-9.

5. Yu P, Huang B, Shen $M$, et al. p15(PAF), a novel PCNA associated factor with increased expression in tumor tissues. Oncogene 2001;20:484-9.

6. Petroziello J, Yamane A, Westendorf L, et al. Suppression subtractive hybridization and expression profiling identifies a unique set of genes overexpressed in non-small-cell lung cancer. Oncogene 2004;23:7734-45.

7. Mizutani K, Onda M, Asaka S, et al. Overexpressed in anaplastic thyroid carcinoma-1 (OEATC-1) as a novel gene responsible for anaplastic thyroid carcinoma. Cancer 2005;103:1785-90.

8. Shi L, Zhang SL, Li K, et al. NS5ATP9, a gene upregulated by HCV NS5A protein. Cancer Lett 2008;259:192-7.

9. Ma F, Zhi C, Wang M, et al. Dysregulated NF-kB signal promotes the hub gene PCLAF expression to facilitate nasopharyngeal carcinoma proliferation and metastasis. Biomed Pharmacother 2020;125:109905.

10. De Biasio A, de Opakua AI, Mortuza GB, et al. Structure of p15(PAF)-PCNA complex and implications for clamp sliding during DNA replication and repair. Nat Commun 2015;6:6439.

11. Yuan RH, Jeng YM, Pan HW, et al. Overexpression of KIAA0101 predicts high stage, early tumor recurrence, and poor prognosis of hepatocellular carcinoma. Clin Cancer Res 2007;13:5368-76.

12. Kais Z, Barsky SH, Mathsyaraja H, et al. KIAA0101 interacts with BRCA1 and regulates centrosome number. Mol Cancer Res 2011;9:1091-9.
13. Jin $\mathrm{C}$, Liu Z, Li Y, et al. PCNA-associated factor P15PAF , targeted by FOXM1, predicts poor prognosis in highgrade serous ovarian cancer patients. Int J Cancer 2018;143:2973-84.

14. Xia J, Gill EE, Hancock RE. NetworkAnalyst for statistical, visual and network-based meta-analysis of gene expression data. Nat Protoc 2015;10:823-44.

15. Chang CN, Feng MJ, Chen YL, et al. p15(PAF) is an Rb/ E2F-regulated S-phase protein essential for DNA synthesis and cell cycle progression. PLoS One 2013;8:e61196.

16. Emanuele MJ, Ciccia A, Elia AE, et al. Proliferating cell nuclear antigen (PCNA)-associated KIAA0101/PAF15 protein is a cell cycle-regulated anaphase-promoting complex/cyclosome substrate. Proc Natl Acad Sci U S A 2011;108:9845-50.

17. Turchi L, Fareh M, Aberdam E, et al. ATF3 and p15PAF are novel gatekeepers of genomic integrity upon UV stress. Cell Death Differ 2009;16:728-37.

18. Xie C, Yao M, Dong Q. Proliferating cell unclear antigenassociated factor (PAF15): a novel oncogene. Int J Biochem Cell Biol 2014;50:127-31.

19. Kato T, Daigo Y, Aragaki M, et al. Overexpression of KIAA0101 predicts poor prognosis in primary lung cancer patients. Lung Cancer 2012;75:110-8.

20. Zhu K, Diao D, Dang C, et al. Elevated KIAA0101 expression is a marker of recurrence in human gastric cancer. Cancer Sci 2013;104:353-9.

21. Colak D, Nofal A, Albakheet A, et al. Age-specific gene expression signatures for breast tumors and cross-species conserved potential cancer progression markers in young women. PLoS One 2013;8:e63204.

22. Shubbar E, Kovács A, Hajizadeh S, et al. Elevated cyclin B2 expression in invasive breast carcinoma is associated with unfavorable clinical outcome. BMC Cancer 2013;13:1.

23. Lv W, Su B, Li Y, et al. KIAA0101 inhibition suppresses cell proliferation and cell cycle progression by promoting the interaction between $\mathrm{p} 53$ and $\mathrm{Sp} 1$ in breast cancer. Biochem Biophys Res Commun 2018;503:600-6.

24. Marie SK, Okamoto OK, Uno M, et al. Maternal embryonic leucine zipper kinase transcript abundance correlates with malignancy grade in human astrocytomas. Int J Cancer 2008;122:807-15.

Cite this article as: Liu J, He Y, Li C, Zhou R, Yuan Q, Hou J, Wu G. Increased KIAA0101 gene expression associated with poor prognosis in breast cancer. Transl Cancer Res 2021;10(9):4009-4019. doi: 10.21037/tcr-20-3064 